\title{
Independency of Fe ions in hemoglobin on immunomagnetic reduction assay
}

\author{
S.Y. Yang ${ }^{\text {a,b }}$, C.B. Lan ${ }^{\text {b }}$, C.H. Chen ${ }^{\text {b }}$, H.E. Horng ${ }^{\text {b,* }}$, Chin-Yih Hong ${ }^{\text {c,* }}$, H.C. Yang ${ }^{\text {d,* }}$, Y.K. Lai ${ }^{\text {e,f }}$, \\ Y.H. Lin ${ }^{\mathrm{g}}$, K.S. Teng ${ }^{\mathrm{g}}$ \\ a MagQu Co. Ltd., Sindian City, Taipei County 231, Taiwan \\ ${ }^{\mathrm{b}}$ Institute of Electro-optical Science and Technology, National Taiwan Normal University, Taipei 116, Taiwan

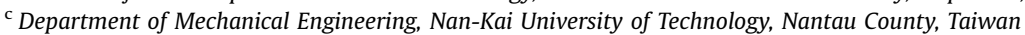 \\ d Department of Physics, National Taiwan University, Taipei 106, Taiwan \\ e College of Life Sciences, National Tsing Hua University, Hsinchu City 300, Taiwan \\ ${ }^{\mathrm{f}}$ Department of Bioresources, Da-Yeh University, Changhua 515, Taiwan \\ ${ }^{\mathrm{g}}$ Apex Biotechnology Co. Ltd., Hsinchu City 300, Taiwan
}

\section{A R T I C L E I N F O}

\section{Article history:}

Received 4 December 2008

Received in revised form

13 March 2009

Available online 12 June 2009

PACS:

75.50.Mm

81.20.-n

Keywords:

Immunomagnetic reduction

Hemoglobin

Magnetic nanoparticle

\begin{abstract}
A B S T R A C T
Immunomagnetic reduction (IMR), which involves measuring the reduction in the ac magnetic susceptibility of magnetic reagents, is due to the association between bio-functionalized magnetic nanoparticles and target bio-molecules. This has been demonstrated for assaying proteins in solutions free of Fe ions, such as serum. In this work, the validity of IMR assay for samples rich in Fe ions like hemoglobin $(\mathrm{Hb})$ is investigated. According to the results, there is no magnetic signal contributed by Fe-ion-rich Hb. Furthermore, the results show a high sensitivity in assaying hemoglobin A1c (HbA1c) by using IMR.
\end{abstract}

(c) 2009 Published by Elsevier B.V.

\section{Introduction}

By bio-functionalizing the surface of magnetic nanoparticles with antibodies, magnetic nanoparticles become useful in diagnosis such as magnetic resonance imaging (MRI) contrast agent [1,2], molecule extraction, or immunoassay [3-6]. Especially in immunoassay, numerous reports show advantages and promising clinical applications when using bio-functionalized magnetic nanoparticles as labeling markers. With the aid of versatile measuring technologies for the magnetic properties of magnetic nanoparticles which are bound to target molecules, the concentrations of target molecules can be determined.

Some groups proposed and demonstrated that Brownian relaxation time becomes longer as bio-functionalized magnetic nanoparticles bind with target bio-molecules, because the hydrodynamic size of nanoparticles becomes larger [7,8]. Thus, by measuring the frequency-dependent magnetic susceptibility of magnetic nanoparticles in liquid, the shift of the peak frequency for imaginary part of ac magnetic susceptibility of magnetic

\footnotetext{
* Corresponding authors.

E-mail addresses: phyfv001@scc.ntnu.edu.tw (H.E. Horng), cyhong@nkut.edu.tw (C.-Y. Hong), hcyang@phys.ntu.edu.tw (H.C. Yang).
}

nanoparticles in liquid due to the binding between nanoparticles and target bio-molecules can be found $[9,10]$. It is further pointed out that the peak-frequency shift is an indicator for detecting the concentration of target bio-molecules in samples. Instead of measuring the frequency-dependent imaginary part of ac magnetic susceptibility, some co-authors recently proposed an alternative methodology, referred to as immunomagnetic reduction (IMR) [11], which involves the measurement of reduction in ac magnetic susceptibility of magnetic reagent due to the association between bio-functionalized magnetic nanoparticles and the target molecules. The assay mechanism of IMR is briefly introduced here. For magnetic reagents, bio-functionalized magnetic nanoparticles are individually dispersed in hydrophilic solution. Under external ac magnetic fields, each of the magnetic nanoparticles can be physically rotated through the magnetic interaction between the applied magnetic fields and magnetic nanoparticles. Thus, the magnetic reagent exhibits an ac magnetic moment, which is usually referred to ac magnetic susceptibility $\chi_{\mathrm{ac}}$. As long as biofunctionalized magnetic nanoparticles associate with target molecules, via antibody-antigen conjugation, associated nanoparticles become either larger or clustered. These larger or clustered magnetic nanoparticles become more immobile compared to un-associated magnetic nanoparticles. This implies that associated magnetic nanoparticles cannot show ac magnetic response 
as well as un-associated magnetic nanoparticles. Hence, the resultant $\chi_{\mathrm{ac}}$ of magnetic reagent would be reduced due to the immobility of magnetic nanoparticles associated with target molecules. Theoretically, when more target molecules bind to magnetic nanoparticles, more immobile nanoparticles are produced; in turn, the reduction in $\chi_{\mathrm{ac}}$ should be larger. Therefore, the concentration of the target molecules in the sample can be determined via measuring the reduction in $\chi_{\mathrm{ac}}$ of the magnetic reagent. The feasibility of assaying proteins, viruses, or chemicals in phosphate buffer saline (PBS) solution or serum has been demonstrated [12-14]. The results reveal such features as low interference, i.e. high specificity, for IMR assays. However, for samples like whole blood, there are magnetic ions, usually referring to Fe ions carried with hemoglobin ( $\mathrm{Hb})$. This may lead to unexpected interference from Fe ions to $\chi_{\mathrm{ac}}$ of the magnetic reagent. In this work, we would like to clarify the influence of Fe ions in hemoglobin on the IMR assays, as well as to investigate the characterizations of assaying $\mathrm{Hb}$ and $\mathrm{HbA} 1 \mathrm{c}$ using IMR.

\section{Experimental details}

The sample (Fujirebio) used here is a PBS solution having $\mathrm{HbA} 1 \mathrm{c}$ of $1 \mathrm{mg} / \mathrm{ml}$ and $\mathrm{Hb}$ with unknown concentration, referred to as $\mathrm{HbA} 1 \mathrm{c} / \mathrm{Hb}$ solution. To detect $\mathrm{Hb}$ and $\mathrm{HbA} 1 \mathrm{c}$, anti-Hb (Cortex, CR8000M) and anti-HbA1c (Good Biotech, D0108-2D) were separately coated onto magnetic nanoparticles. The magnetic nanoparticles coated with anti-Hb or anti-HbA1c are denoted as $\mathrm{Hb} / \mathrm{HbA} 1 \mathrm{c}$ reagent, respectively. The magnetic nanoparticles are $\mathrm{Fe}_{3} \mathrm{O}_{4}$ with dextran as outmost layer (MagQu Co. Ltd.). Anti-Hb and anti-HbA1c were covalently bound with dextran [6]. The magnetic nanoparticles have a mean diameter of $67 \mathrm{~nm}$. For the measurement of $\chi_{\mathrm{ac}}$, a magnetosusceptometer (MagQu Co. Ltd., XacPro-E101) was used in the experiment. Reagents and samples were contained in a glass tube and loaded into the magnetosusceptometer for the detection of $\chi_{\mathrm{ac}}$ as a function of time.

\section{Results and discussion}

First of all, the effect of the magnetic property of Fe ions in $\mathrm{Hb}$ on $\chi_{\text {ac }}$ was examined. To do this, an empty glass tube was loaded into the magnetosusceptometer. The time-dependent signal for $\chi_{\mathrm{ac}}$ is around 8.09, as shown in Fig. 1 for the first $1 \mathrm{~h}$. This means the background noise level of the magnetosusceptometer is around 8.09. Then, $40 \mu \mathrm{l}$ PBS solution was pipetted into the glass tube, followed by measuring $\chi_{\mathrm{ac}}$ signal for $1 \mathrm{~h}$. The data are shown in Fig. 1 for the time interval from 1 to $2 \mathrm{~h}$, and shows the same amplitude as that of glass tube. It was found that PBS does not contribute any magnetic signal to $\chi_{\mathrm{ac}}$. Finally, $60 \mu \mathrm{l} \mathrm{HbA1c/Hb}$ solution was added into the $40 \mu \mathrm{l}$ PBS solution in the glass tube. The $\chi_{\text {ac }}$ signal was instantly recorded for another $2 \mathrm{~h}$, as plotted with data points in Fig. 1 from 2 to $4 \mathrm{~h}$. The results in Fig. 1 show the fact that there is no difference in the amplitudes of $\chi_{\mathrm{ac}}$ for the empty glass, $40 \mu \mathrm{l}$ PBS solution, and $60 \mu \mathrm{l} \mathrm{HbA1c}$ solution. It is clear that $\mathrm{Fe}$ ions in $\mathrm{Hb}$ or $\mathrm{HbA1c}$ do not contribute to the value of $\chi_{\mathrm{ac}}$.

On the other hand, $1 \mathrm{~h}$ later after we injected $60 \mu$ ldiluted $\mathrm{HbA} 1 \mathrm{c} / \mathrm{Hb}$ solution of $1: 1000$, i.e. $1 \mu \mathrm{g}-\mathrm{HbA} 1 \mathrm{c} / \mathrm{ml}$, into another empty glass tube, followed by pipetting $40 \mu \mathrm{l} \mathrm{Hb}$ reagent into the glass tube, the $\chi_{\text {ac }}$ signal abruptly increased from 8.09 to 51 , as shown in Fig. 2. Obviously, the high amplitude $(\sim 51)$ for $\chi_{\mathrm{ac}}$ is contributed by magnetic nanoparticles in $\mathrm{Hb}$ reagent. This

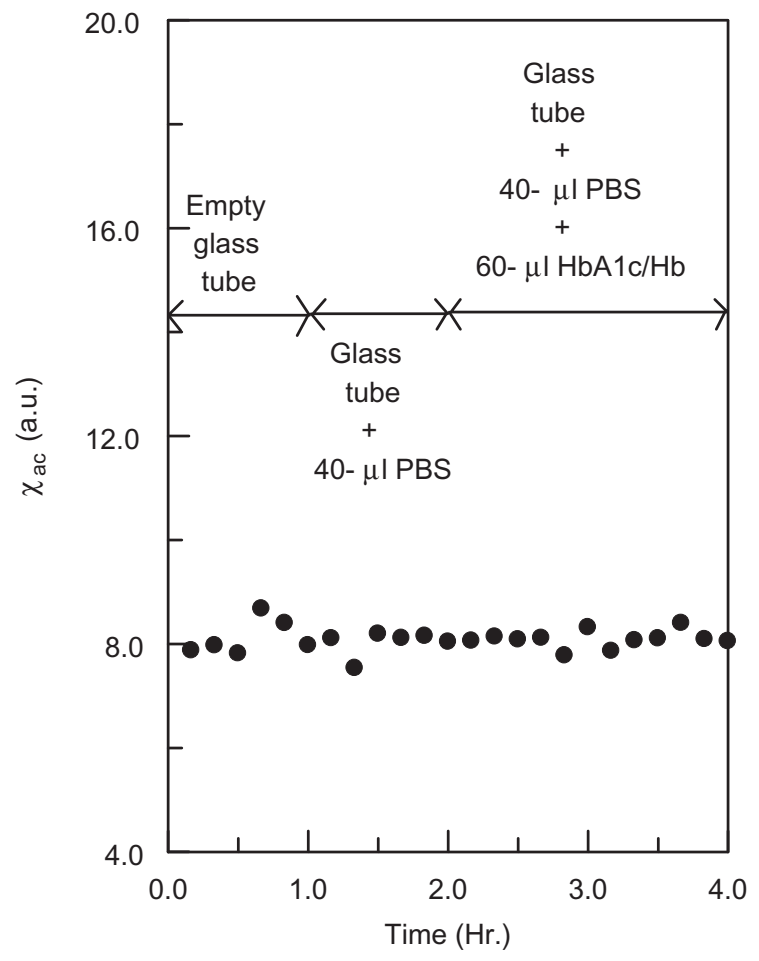

Fig. 1. Time-dependent $\chi_{\mathrm{ac}}$ signal for an empty glass tube (from 0 to $1 \mathrm{~h}$ ), $40 \mu \mathrm{l}$ PBS solution in the glass tube (from 1 to $2 \mathrm{~h}$ ), and mixture of $40 \mu \mathrm{l}$ PBS solution and $60 \mu \mathrm{l} \mathrm{HbA} 1 \mathrm{c} / \mathrm{Hb}$ solution having HbA1c of $1 \mathrm{mg} / \mathrm{ml}$ (from 2 to $4 \mathrm{~h}$ ).

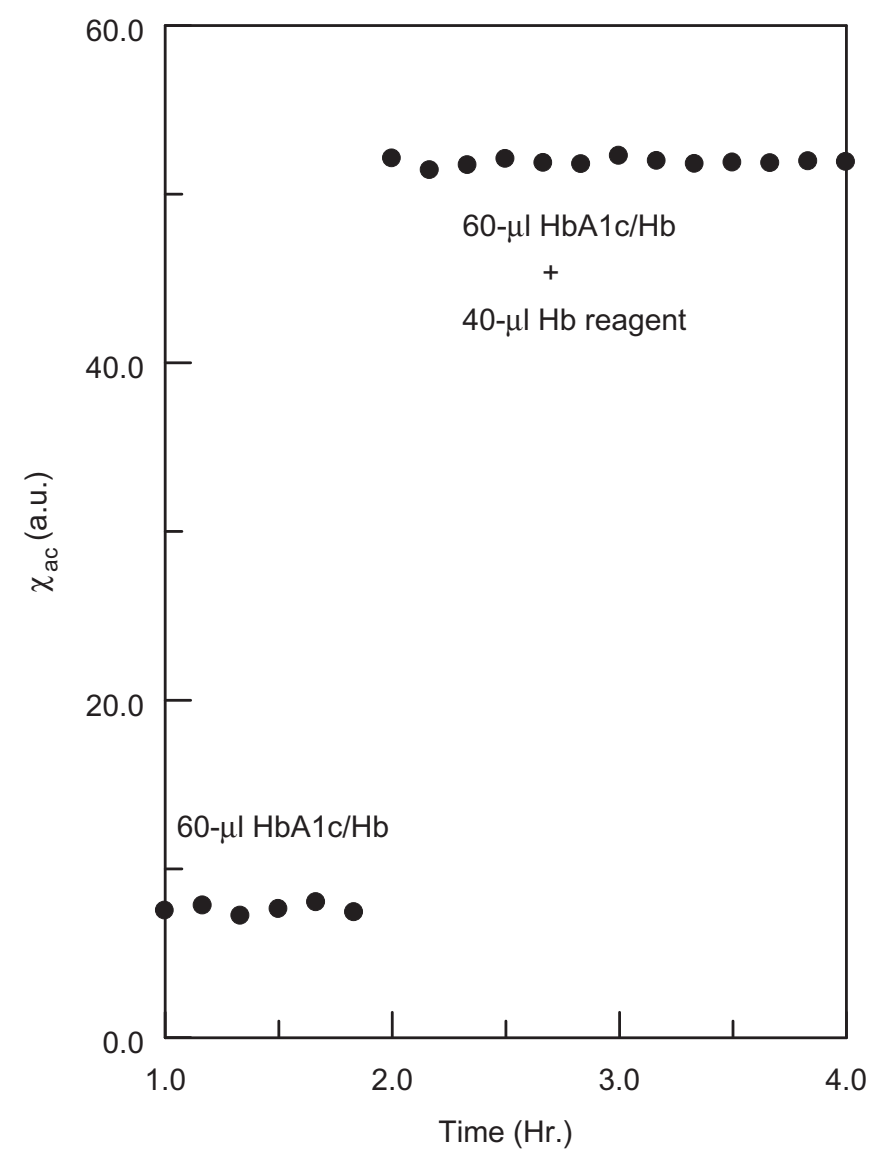

Fig. 2. Time-dependent $\chi_{\mathrm{ac}}$ signal for $60 \mu \mathrm{l}$ diluted $\mathrm{HbA} 1 \mathrm{c} / \mathrm{Hb}$ solution having $1 \mu \mathrm{g} / \mathrm{ml} \mathrm{HbA} 1 \mathrm{c}$ in a glass tube (from 0 to $1 \mathrm{~h}$ ), and the mixture of $40 \mu \mathrm{l} \mathrm{Hb}$ reagent and the diluted blood in the glass tube. 


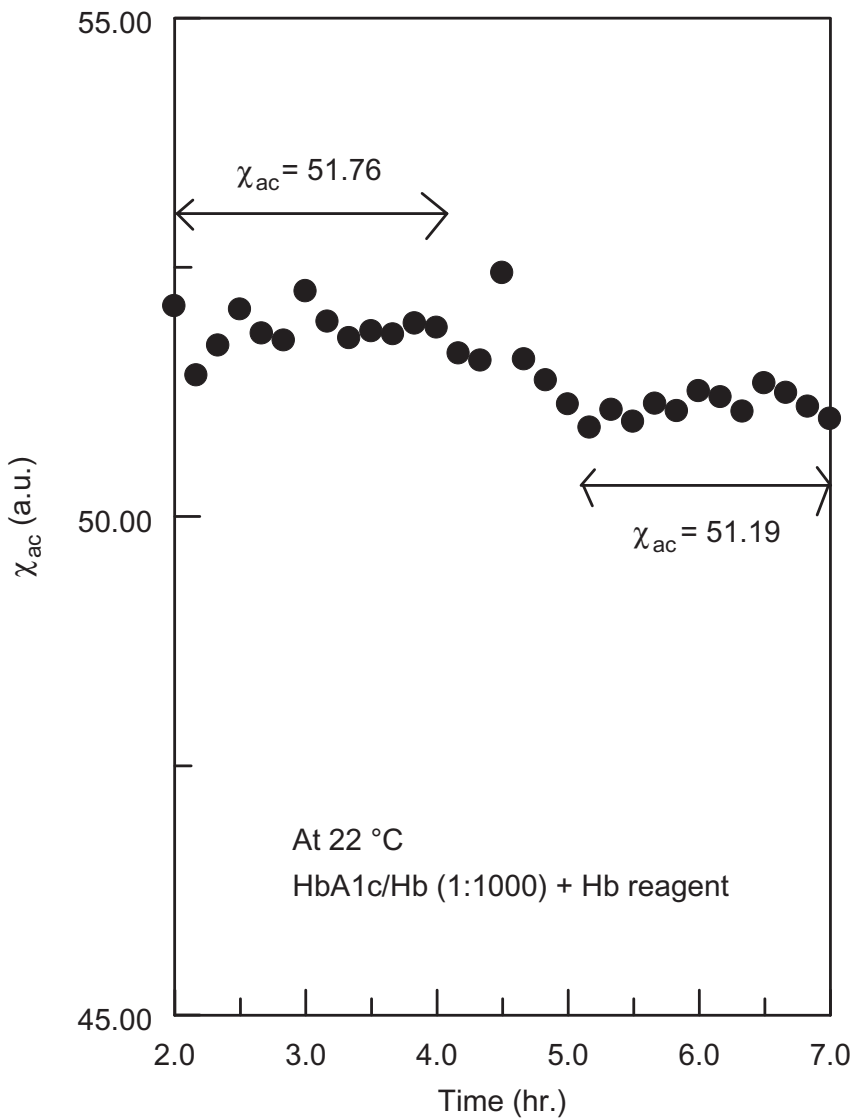

Fig. 3. $\chi_{\mathrm{ac}}$ signal of $\mathrm{Hb}$ reagent as a function of time after the addition of $60 \mu \mathrm{l}$ $1 \mu \mathrm{g}-\mathrm{HbA} 1 \mathrm{c} / \mathrm{ml} \mathrm{HbA1c/Hb}$ solution into $40 \mu \mathrm{l} \mathrm{Hb}$ reagent.

evidences the rotation of magnetic nanoparticles in reagent under the action of external ac magnetic fields. The time-dependent $\chi_{\mathrm{ac}}$ of the mixture of reagent and $\mathrm{HbA1c} / \mathrm{Hb}$ solution was kept for further detection.

Few hours later, a significant reduction in $\chi_{\mathrm{ac}}$ of the mixture, $\mathrm{Hb}$ reagent and diluted $\mathrm{HbA} 1 \mathrm{c} / \mathrm{Hb}$ solution, was observed, as shown in Fig. 3. $\chi_{\text {ac }}$ starts to decrease at the 4 th hour, and then reaches another constant at the 5 th hour. The decreasing $\chi_{\mathrm{ac}}$ from the 4th to 5th hour reveals that anti-Hb functionalized magnetic nanoparticles are conjugating with $\mathrm{Hb}$ in the diluted $\mathrm{HbA} 1 \mathrm{c} / \mathrm{Hb}$ solution. This conjugation is finished at the 5th hour, and $\chi_{\text {ac }}$ becomes fixed. Due to the conjugation, some magnetic nanoparticles in the reagent become more immobile, which accounts for the reduction in $\chi_{\mathrm{ac}}$ of the mixture. According to Fig. 3 , the reduction was found to be $1.10 \%$.

The results in Fig. 3 are from a single test for the diluted $\mathrm{HbA} 1 \mathrm{c} / \mathrm{Hb}$ solution $(1: 1000$, i.e. $1 \mu \mathrm{g}-\mathrm{HbA} 1 \mathrm{c} / \mathrm{ml})$. Another two tests were also done for the same sample. The data from these three tests give an average of $1.08 \%$ and a standard deviation of $0.04 \%$ for the reduction in $\chi_{\mathrm{ac}}$, i.e. $\mathrm{CV}=3.70 \%$ for the $\chi_{\mathrm{ac}}$-reduction measurement, shown in Fig. 3.

In addition to assaying $\mathrm{Hb}$, the $\mathrm{HbA} 1 \mathrm{c}$ in the $1: 1000$ diluted $\mathrm{HbA1c/Hb}$ solution is examined by using the HbA1c reagent. The significant reduction in $\chi_{\mathrm{ac}}$ of the $\mathrm{HbA} 1 \mathrm{c}$ reagent was observed after mixing the diluted $\mathrm{HbA} 1 \mathrm{c} / \mathrm{Hb}$ solution with the HbA1c reagent. This fact evidences the feasibility of detecting HbA1c using IMR. According to the three tests assaying HbA1c for $1: 1000$ diluted $\mathrm{HbA} 1 \mathrm{c} / \mathrm{Hb}$, values of $0.88 \%$ and $0.03 \%$ were obtained for the average and standard deviation of the reduction in $\chi_{\mathrm{ac}}$ of the $\mathrm{HbA1c}$ reagent, respectively, as plotted in Fig. 4.

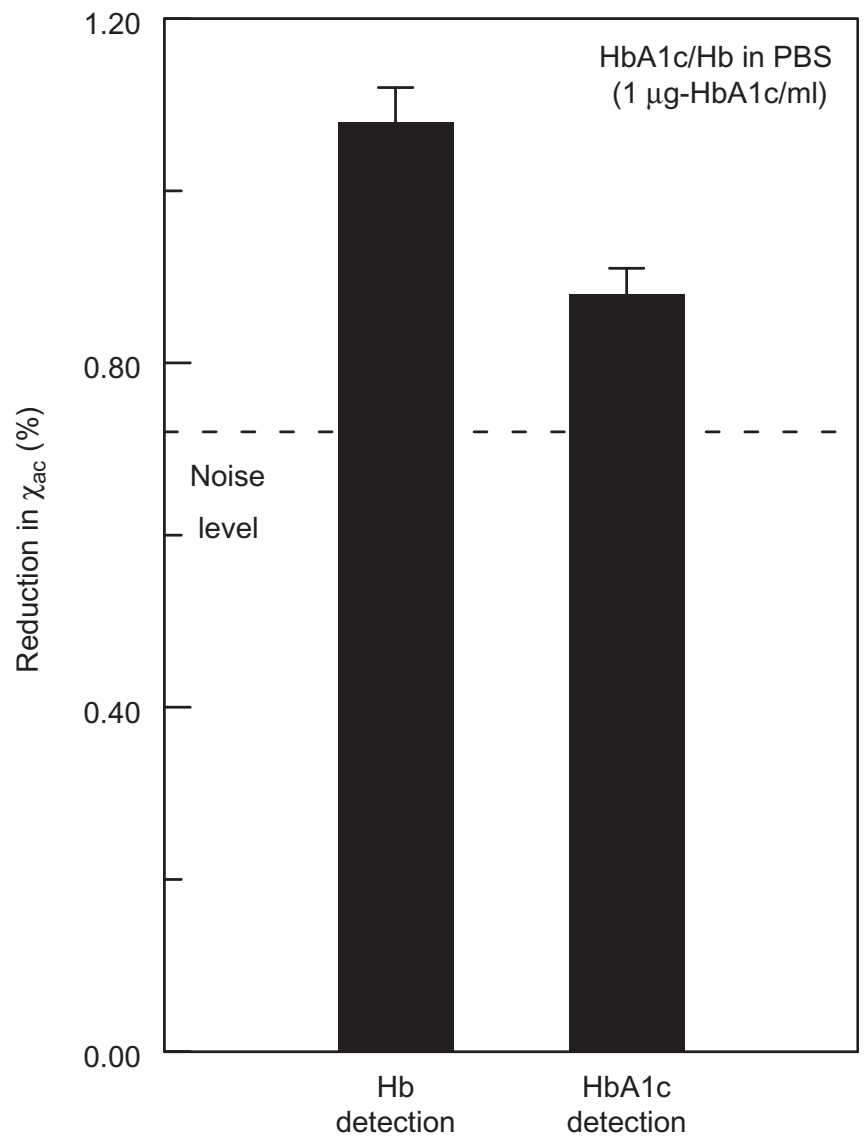

Fig. 4. Resultant reductions (in percentage) in the $\chi_{\mathrm{ac}}$ of $\mathrm{Hb} / \mathrm{HbA} 1 \mathrm{c}$ reagents for triply detecting $\mathrm{Hb} / \mathrm{HbA} 1 \mathrm{c}$ in PBS solution.

\section{Conclusion}

It is evidenced that there is no observable contribution from $\mathrm{Fe}$ ions in hemoglobin to the detected signals for IMR assay. This fact reveals the possibility to use whole blood as tested samples for IMR. Such merit is very important to promote IMR to point-of-care test (e.g. at-home detection), in which whole blood is the only candidate, neither serum nor plasma. Besides, the results show the promising feasibility of detecting hemoglobin or degree of blood sugar, i.e. the concentration ratio of HbA1c to $\mathrm{Hb}$, using IMR. However, more efforts are to be paid for clinical trials in the future.

\section{Acknowledgement}

This work is supported by the National Science Council of Taiwan under Grant numbers NSC97-2112-M-003-012 and NSC98-2752-M-002-016-PAE, and by Ministry of Economic Affairs of Taiwan under Grant numbers 1Z970688 (SBIR) and S09800226-203 (JAID).

\section{References}

[1] D. Roberts, W.L. Zhu, C.M. Frommen, Z. Osenzweig, J. Appl. Phys. 87 (2000) 6208.

[2] D.E. Sosnovik, E.A. Schellenberger, M. Nahrendorf, M.S. Novikov, T. Matsui, G. Dai, F. Reynolds, L. Grazette, A. Rosenzweig, R. Weissleder, L. Josephson, Magn. Reson. Med. 54 (2005) 718.

[3] R. Kötitz, W. Weitschies, L. Trahms, W. Brewer, W. Semmler, J. Magn. Magn. Mater. 194 (1999) 62. 
[4] S.K. Lee, W.R. Myers, H.L. Grossman, H.-M. Cho, Y.R. Chemla, J. Clarke, Appl. Phys. Lett. 81 (2002) 3094.

[5] K. Enpuku, T. Minotani, T. Gima, Y. Kuroki, Y. Itoh, M. Yamashita, Y. Katakura, S. Kuhara, Jpn. J. Appl. Phys. 38 (1999) L1102.

[6] H.C. Yang, S.Y. Yang, S.H. Liao, G.L. Fang, W.H. Huang, C.H. Liu, H.E. Horng, C.Y. Hong, J. Appl. Phys. 99 (2006) 124701.

[7] R. Kötitz, P.C. Fannin, L. Trahms, J. Magn. Magn. Mater. 149 (1995) 42.

[8] P.C. Fannin, B.K.P. Scaife, S.W. Charles, J. Magn. Magn. Mater. 72 (1988) 95.

[9] S.H. Chung, A. Hoffmann, S.D. Bader, C. Liu, B. Kay, L. Makowski, L. Chen, Appl. Phys. Lett. 85 (2004) 2971.
[10] S.H. Chung, A. Hoffmann, K. Guslienko, S.D. Bader, C. Liu, B. Kay, L. Makowski, L. Chen, J. Appl. Phys. 97 (2005) 10R101.

[11] C.Y. Hong, C.C. Wu, Y.C. Chiu, S.Y. Yang, H.E. Horng, H.C. Yang, Appl. Phys. Lett. 88 (2006) 212512.

[12] C.Y. Hong, W.H. Chen, Z.F. Jian, S.Y. Yang, H.E. Horng, L.C. Yang, H.C. Yang, Appl. Phys. Lett. 90 (2007) 74105.

[13] S.Y. Yang, J.J. Chieh, W.C. Wang, C.Y. Yu, C.B. Lan, J.H. Chen, H.E. Horng, C.Y. Hong, H.C. Yang, J. Virol. Methods 153 (2008) 250.

[14] S.Y. Yang, Z.F. Jian, J.J. Chieh, H.E. Horng, H.C. Yang, C.Y. Hong, J. Virol. Methods 149 (2008) 334. 